Supporting Information

for

\title{
3D-to-3D Microscale Shape-Morphing from Configurable Helices with Controlled Chirality
}

Zhenyu Zhao, Yisheng He, Xiao Meng, Chunhong Ye*

School of Physical Science and Technology, ShanghaiTech University, Shanghai, 201210, P. R. China

Email: yechh@shanghaitech.edu.cn 


\section{Theoretical analysis of upward/downward rolling behavior of PNIPAm/PMMA}

microsheet. PNIPAm layer has significantly swelling behavior when the temperature is below the LCST. While, PMMA keeps constant volume as passive layer against the swelling of PNIPAm. Therefore, mismatched interfacial force is generated between the two layers which triggers microsheets self-rolling upwards. On the other hand, PNIPAm is hydrophobic and deswells as increasing temperature above its LSCT. The shrink of PNIPAm layer induces downwards bending to form a micro-helical morphology again. The upward/downward rolling behaviors can be qualitatively analyzed with the curvature of micro-helices at corresponding temperatures.

The PNIPAm/PMMA microsheet can be considered as microscopic elastic plate. The curvature of microsheet can be estimated by Timoshenko equation as reported previously, ${ }^{1,2}$

$$
\kappa=\frac{6\left(\varepsilon_{2}-\varepsilon_{1}\right)(1+m)^{2}}{h\left(3(1+m)^{2}+(1+m n)\left(m^{2}+\frac{1}{m n}\right)\right)}
$$

Where $\kappa$ is the bending curvature of bilayer structure, $m$ is the thickness ratio of passive layer to active layer, $\mathrm{n}$ is the modulus ratios of PMMA to PNIPAm. $\varepsilon_{2}$ and $\varepsilon_{1}$ are the strains of PNIPAm and PMMA, respectively. $h$ is the total thickness of the bilayer film. Timoshenko equation demonstrates that the curvature is not only determined by swelling ratio and thickness ratio, but also controlled by the ratio of modulus, especially for thermal responsive hydrogels. According to previous reported work, the modulus of PNIPAm increases an order of magnitude when temperature is above the $\mathrm{LCST}^{3}$, which can induce a significant deformation of PNIPAm film even with a small change of volume at the temperature above LCST.

Here, we used PNIPAm/PMMA bilayer with an original thickness of $860 \mathrm{~nm} / 350 \mathrm{~nm}$ as an 
example for calculating the curvature to explain the upward and downward rolling behaviors at $10^{\circ} \mathrm{C}$ and $40{ }^{\circ} \mathrm{C}$. The moduli of PNIPAm were $15 \mathrm{MPa}$ at $10{ }^{\circ} \mathrm{C}$ and $250 \mathrm{MPa}$ at $40{ }^{\circ} \mathrm{C}$ according to previously reported literature. ${ }^{3}$ The swelling ratio of PNIPAm reaches to $150 \%$ at $10{ }^{\circ} \mathrm{C}$ and only $5 \%$ at $40{ }^{\circ} \mathrm{C}$. According to Timoshenko equation, the value of curvature is 69 $\mathrm{mm}^{-1}$ at $10{ }^{\circ} \mathrm{C}$, which is close to the experimental result $\left(76 \pm 3 \mathrm{~mm}^{-1}\right)$. While the curvature of bilayer is $-41 \mathrm{~mm}^{-1}$ at $40{ }^{\circ} \mathrm{C}$, which is also in the same magnitude as compared with the experiment result $\left(-98 \pm 4 \mathrm{~mm}^{-1}\right)$. The theoretical analysis indicates upward/downward rolling behaviors at temperatures below and above LCST of PNIPAm, respectively, which is in good trend with experimental results. It is worth to notice that the equation is originally derived to explain the bending of bimetal beans upon change of temperature and developed for small deformation. By applying it in the field of shape-morphing polymer film, the swelling degree of polymer is used to substitute the thermal expansion coefficient. And it is only able to provide qualitative not quantitative analysis of the curvature in current case.

The effect of uncollimated UV light on the lateral cross-linkable shadow region. The UV exposure setup used here is demonstrates as Figure S1a. The photomask is tightly contacted with the samples. And the UV light source is a tube-like lamp with filter. A black rectangle box with dimension of $1 \mathrm{~cm} \times 1 \mathrm{~cm} \times 3 \mathrm{~cm}$ was used between the lamp and photomask to stop UV light from every direction. Considering the geometry of the box, the largest incidence angle $(\theta)$ of UV light as shown in Figure $\mathrm{S} 1 \mathrm{~b}$ and $\mathrm{c}$ is around $25.5^{\circ}$. This large incidence angle enables a lateral cross-linkable shadow region. The dimension of this shadow region, $\delta$, from each edge of open slots is determined by incident angle $(\theta)$ and film thickness $(h), \delta \approx 2 h \cdot \tan \theta,{ }^{4}$ which is close to the film thickness according to the custom UV exposure setup used here. The lateral 
size of shadow region is smaller than that of "closed" region on photomask. We hypothesis that the refraction of UV light from silicon substrate also significantly contributed to crosslink the entire lateral dimension of the film under chrome patterned areas.

\section{Supplementary Figures S1-S7}
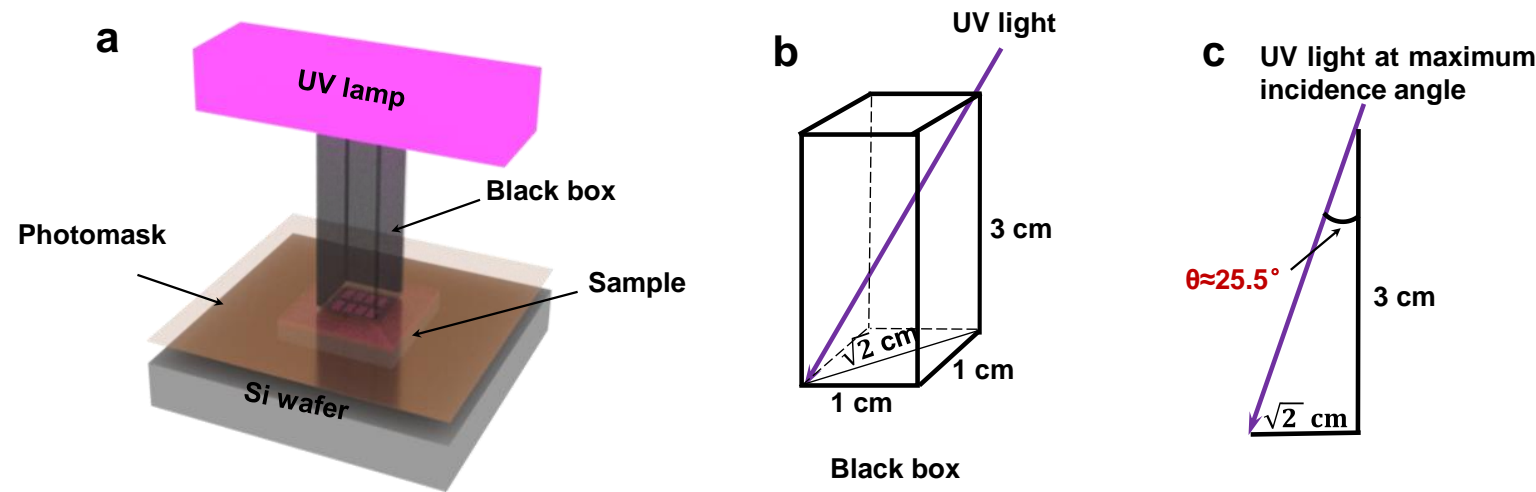

Figure S1. (a) Schematic illustration of custom-designed UV crosslinking setup; (b) The geometry and dimension of black box used between UV lamp and photomask for confining the light travelling path; (c) The estimated maximum incidence angle of UV light based on the longest travelling distance confined by the size of black box.

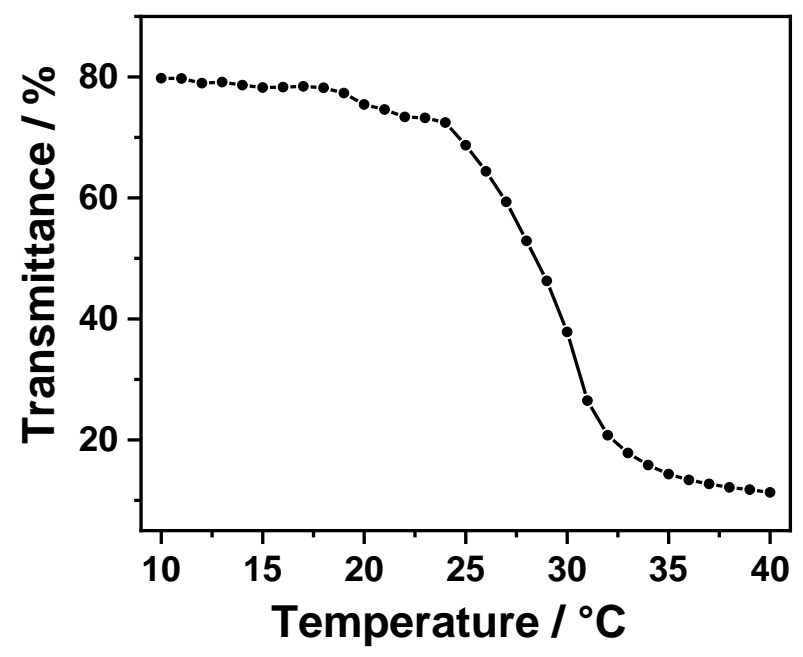


Figure S2. Temperature dependence of the light transmittance through $0.5 \mathrm{mg} / \mathrm{mL}$ PNIPAm aqueous solution.

a
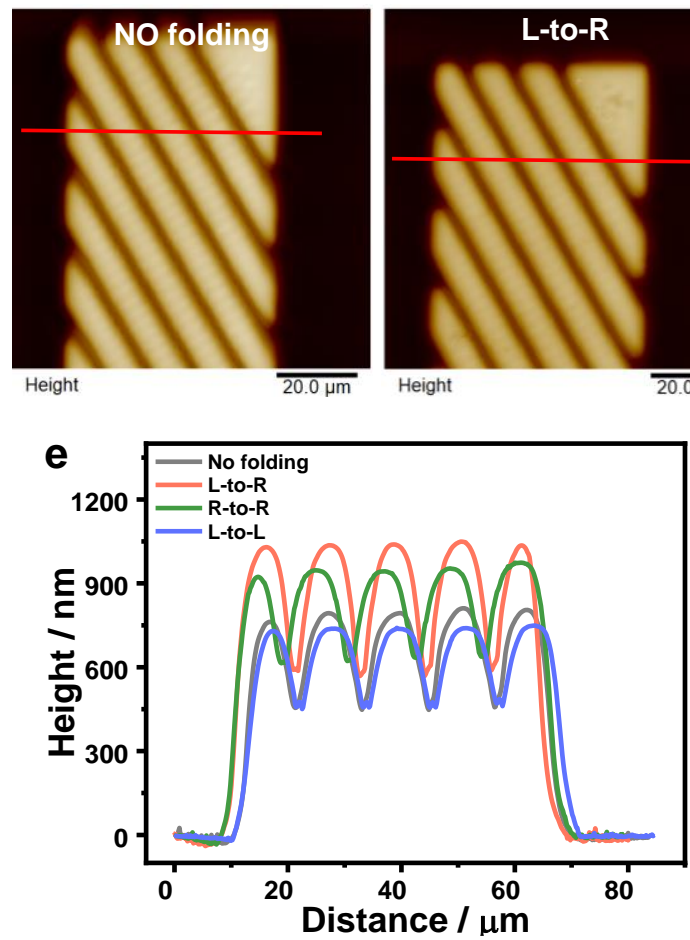

C

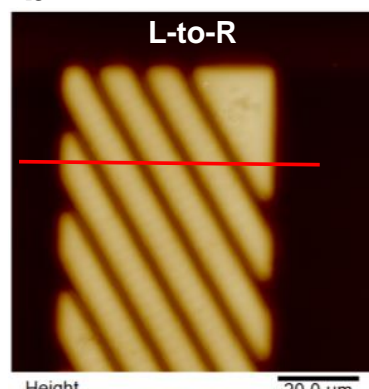

$\overline{20.0 \mu \mathrm{m}}$

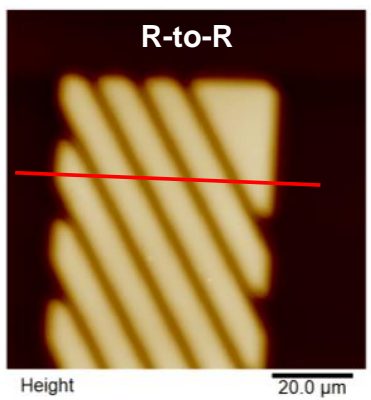

d
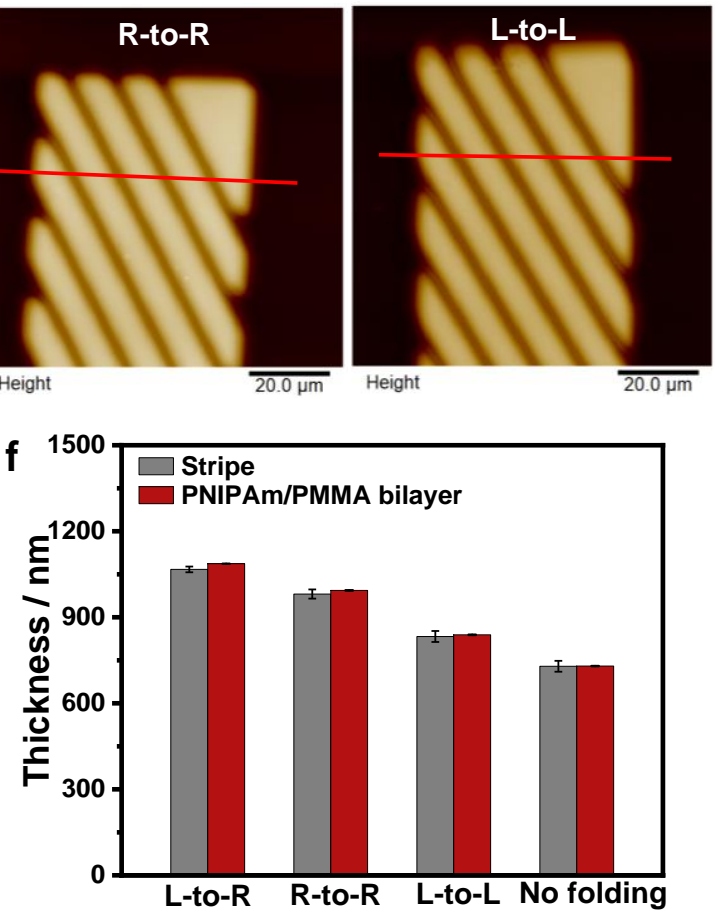

Figure S3. AFM topography images of microsheets with varied shape transformation behaviors (a-d); and (e) corresponding AFM cross-section analysis obtained from the locations marked with red line on the above four microsheets, z-scale: $1.2 \mu \mathrm{m}(\mathrm{a}, \mathrm{d}), 1.7 \mu \mathrm{m}(\mathrm{b}), 1.5 \mu \mathrm{m}$ (c); (f)The comparison of heights of stripes with the total thickness of PNIPAm and PMMA bilayer, the former ones were obtained from AFM height analysis and the latter ones were obtained from ellipsometer measurements. 


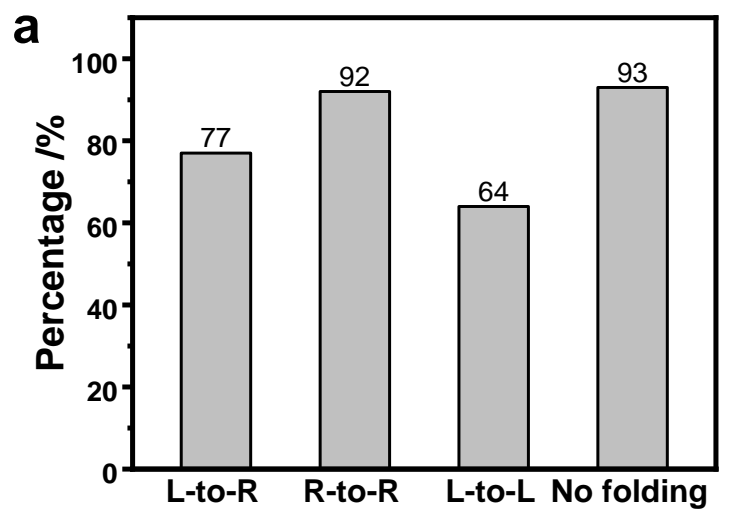

The repeatability of shape reconfiguration

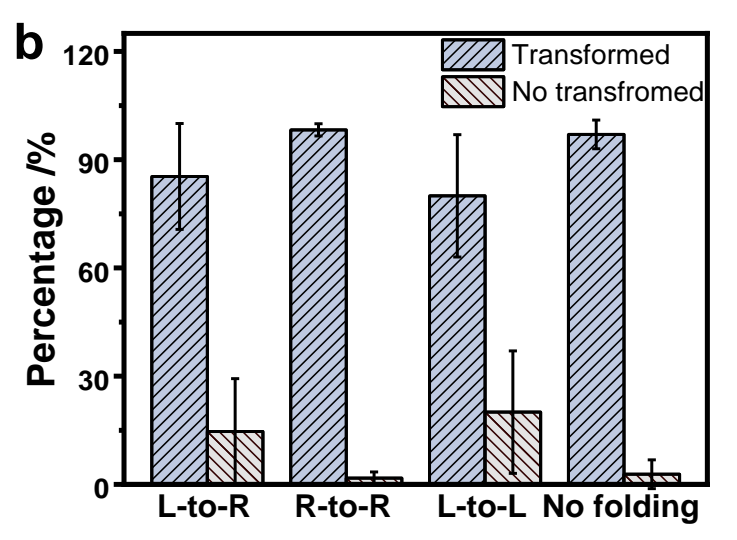

The uniformity of shape reconfiguration

Figure S4. (a) Statistical analysis on the repeatability of samples with the same type of shape reconfiguration in each region. (b) Statistical analysis on uniformity of shape reconfiguration of microsheets from one sample located at each region.
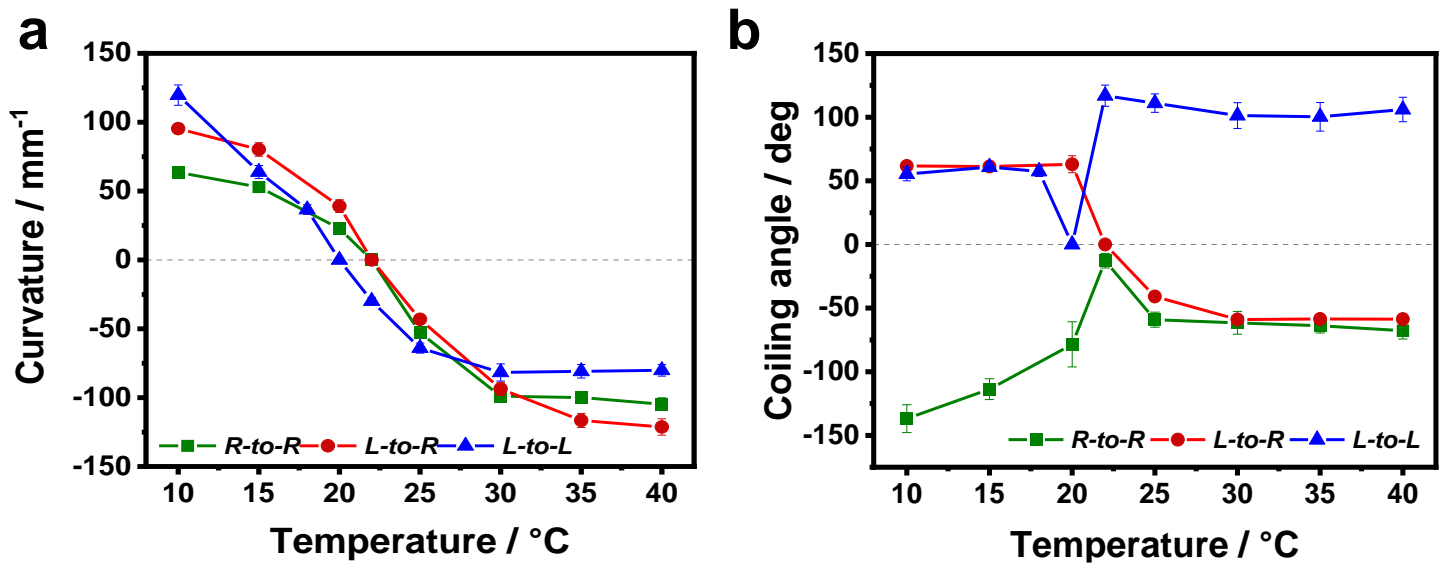

Figure S5. Curvature (a) and coiling angle (b) of micro-helices with varied chirality switch behaviors in the temperature range from $10{ }^{\circ} \mathrm{C}$ to $40{ }^{\circ} \mathrm{C}$. 


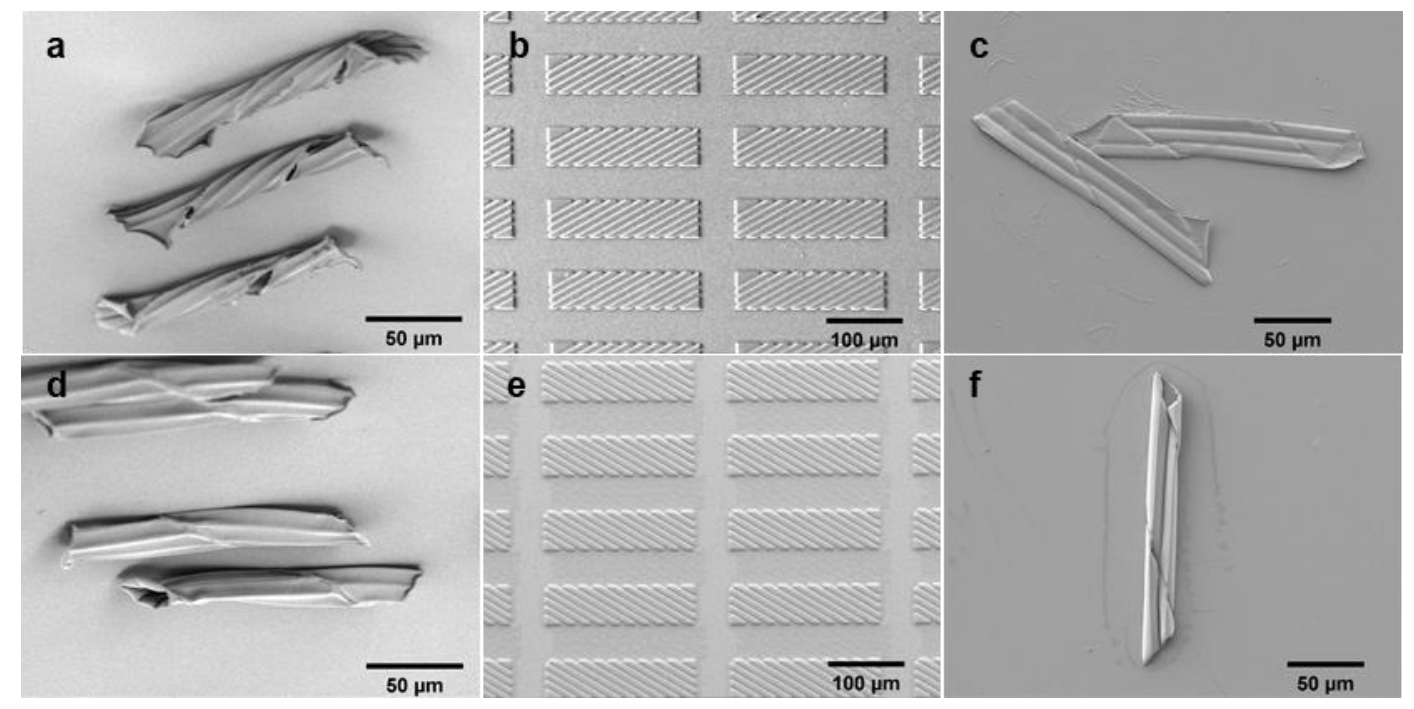

Figure S6. (a) SEM image of freeze-dried chiral structures rolled below LCST from of $30^{\circ}$ stripe-patterned microsheet; (b) SEM image of $30^{\circ}$ stripe-patterned microsheets; (c) SEM image of dried chiral structures rolled above LCST from of $30^{\circ}$ stripe-patterned microsheets; (d) SEM image of freeze-dried chiral structures rolled below LCST from $-30^{\circ}$ stripe-patterned microsheets; (e) SEM image of $-30^{\circ}$ stripe-patterned microsheets; (f) SEM image of dried chiral structures rolled above LCST from $-30^{\circ}$ stripe-patterned microsheet. PNIPAm/PMMA film thickness: $810 \pm 15 \mathrm{~nm} / 375 \pm 7 \mathrm{~nm}$ in (a-c), and $850 \pm 15 \mathrm{~nm} / 371 \pm 9 \mathrm{~nm}$ in (d-f). 

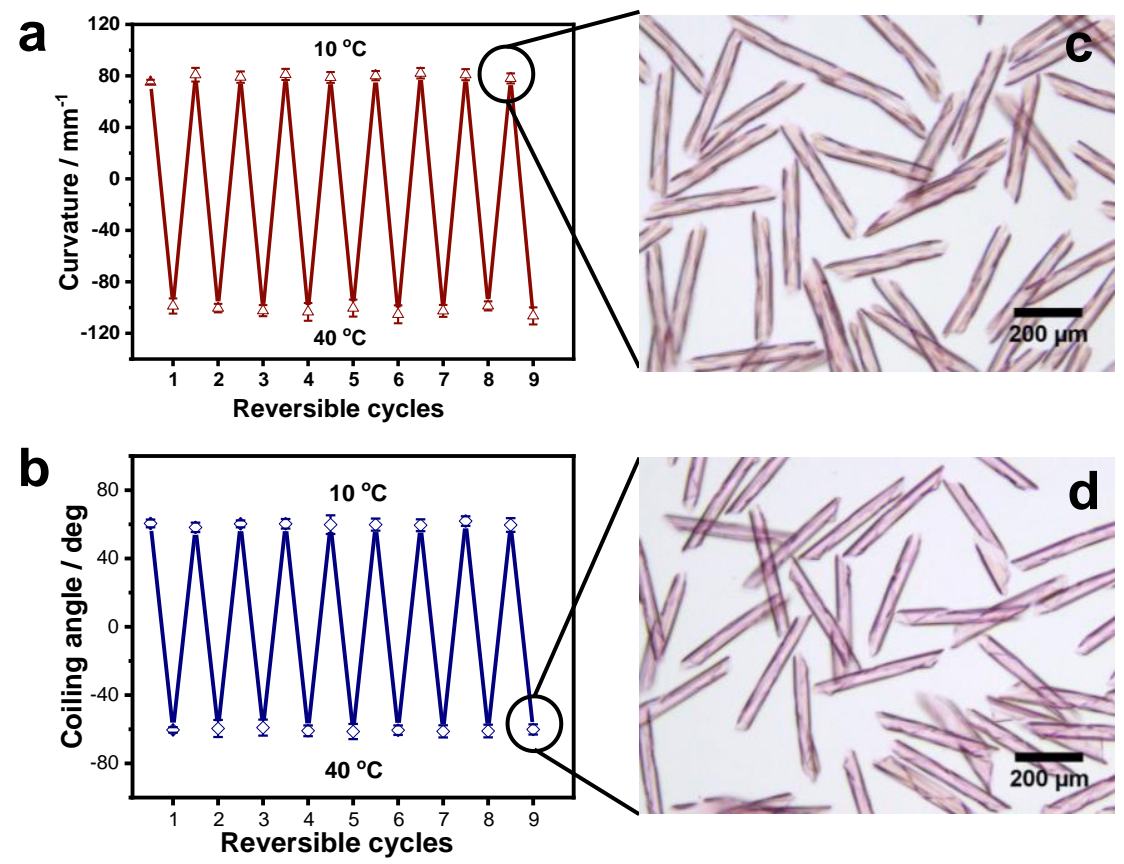

Figure S7. Reversibility of micro-helices at alternating temperatures. Curvature (a) and coiling angle (b) of reversible changed micro-helices morphologies as temperature switching between $10^{\circ} \mathrm{C}$ and $40^{\circ} \mathrm{C}$; (c) Optical microscopy image of micro-helices at $10^{\circ} \mathrm{C}$ after nine cycles; (d) Optical microscopy image of micro-helices at $40{ }^{\circ} \mathrm{C}$ after nine cycles.

a

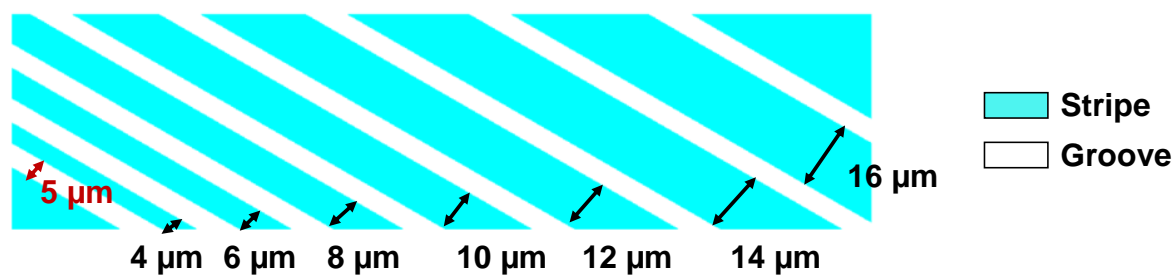

b

b

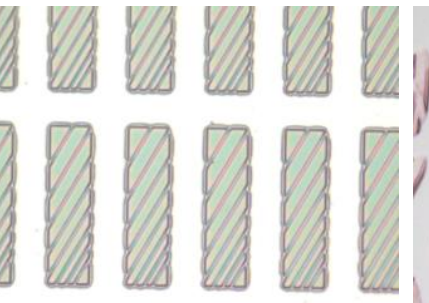

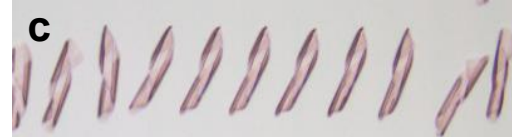

d

D.

2989898181

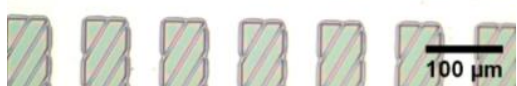

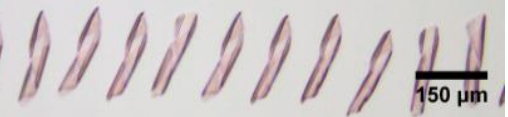

Figure S8. Self-rolling behavior of microsheets with a gradient increased width of stripes. (a) 
Schematic illumination of microsheets with a fix with of groove and a gradient increased width of stripes; (b) Optical microscopy images of microsheets with a gradient increased width; (c) Optical microscopy images of self-rolled micro-helices from microsheets in panel $b$ indicated a gradient of curvature; (d) 3D confocal images of micro-helices as demonstrated in panel c.

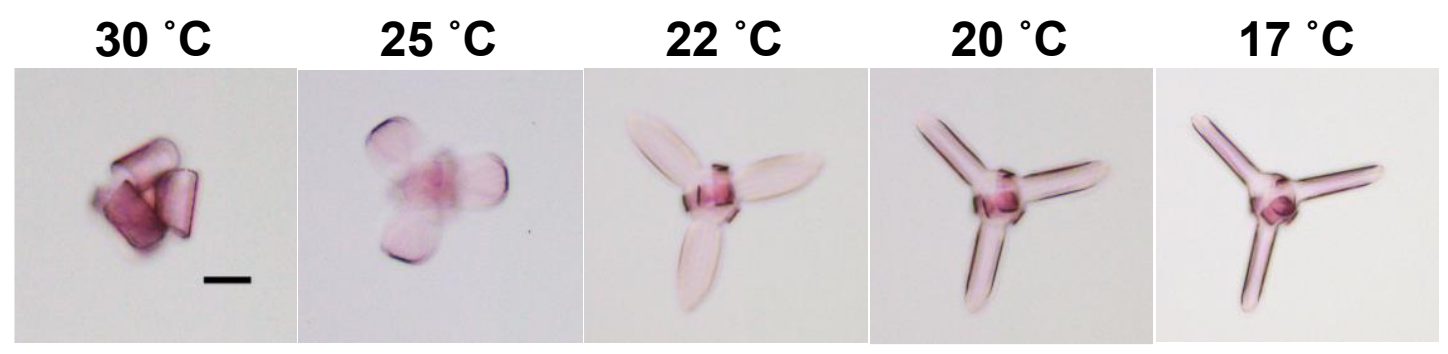

Figure S9. Optical images of biomimetic "orchid" blossoming as temperature varied from $30{ }^{\circ} \mathrm{C}$ to $17^{\circ} \mathrm{C}$. Scale bar: $50 \mu \mathrm{m}$.

\section{Supplementary Movies}

Movie S1. Microhelices with right-to-left handedness switching as temperature decreased from $40{ }^{\circ} \mathrm{C}$ to $10{ }^{\circ} \mathrm{C}$ (stripes oriented with a tilting angle of $30^{\circ}$ ).

Movie S2. Microhelices with right-to-right handedness switching as temperature decreased from $40{ }^{\circ} \mathrm{C}$ to $10^{\circ} \mathrm{C}$ (stripes oriented with a tilting angle of $30^{\circ}$ ).

Movie S3. Microhelices with left-to-left handedness switching as temperature decreased from $40{ }^{\circ} \mathrm{C}$ to $10{ }^{\circ} \mathrm{C}$ (stripes oriented with a tilting angle of $30^{\circ}$ ). 
Movie S4. Microhelices with left-to-right handedness switching as temperature decreased from $40{ }^{\circ} \mathrm{C}$ to $10{ }^{\circ} \mathrm{C}$ (stripes oriented with a tilting angle of $-30^{\circ}$ ).

Movie S5. Reversibility of micro-helices with 9 cycles.

Movie S6. "T-crossing" to "Windmill" 3D reconfiguration.

Movie S7. "Cylinder" to "Scroll" 3D reconfiguration.

Movie S8. Biomimetic blossoming of an "orchid". 
[1] Timoshenko, S. Analysis of Bi-mental Thermostats. J. Opt. Soc. Am. 1925, 11, 233-255.

[2] Stroganov, V.; Pant, J.; Stoychev, G.; Janke, A.; Jehnichen, D.; Fery, A.; Handa, H.; Ionov, L. 4D Biofabrication: 3D Cell Patterning Using Shape-Changing Films. Adv. Funct. Mater. 2018, 28, 1706248.

[3] Hippler, M.; Blasco, E.; Qu, J.; Tanaka, M.; Barner-Kowollik, C.; Wegener, M.; Bastmeyer, M. Controlling the Shape of 3D Microstructures by Temperature and Light. Nat. Commun. 2019, 10, 232.

[4] Bae, J.; Na, J-H.; Santangelo, C. D.; Hayward, R. C. Edge-Defined Metric Buckling of Temperature-Responsive Hydrogel Ribbons and Rings. Polymer 2014, 55, 5908-5914. 